\title{
Mesenchymal Stem Cells Mitigate Cirrhosis through BMP7
}

\author{
Bing $\mathrm{Li}^{\mathrm{a}}$ Qing Shao ${ }^{\mathrm{a}}$ Dong Jia Fan Lia Guofeng Chen ${ }^{\mathrm{a}}$ \\ aLiver Fibrosis Diagnosis and Treatment Center, 302 Hospital of PLA, Beijing China
}

\section{Key Words}

Cirrhosis • Mesenchymal stem cells (MSCs) $\bullet \mathrm{CCl}_{4} \cdot$ Transforming growth factor $\beta 1$ (TGF $\beta 1$ ) Bone morphogenic protein 7 (BMP7)

\begin{abstract}
Background/Aims: Transplantation of mesenchymal stem cells (MSCs) has therapeutic effects on various diseases, while its effect on developing cirrhosis as well as the underlying mechanism remained largely unknown. Methods: Twenty C57BL/6 mice were randomly separated into 2 groups of ten each. One group received transplantation of MSCs, while the other group received saline as control. The mice then received intraperitoneal injection of carbon tetrachloride $\left(\mathrm{CCl}_{4}\right)$ twice per week for 8 weeks to develop cirrhosis. After another 4 weeks, the levels of cirrhosis in these mice were evaluated by liver fibrosis area, portal pressure, sodium balance and excretion. Transcripts of transforming growth factor $\beta 1$ (TGF $\beta 1$ ) and bone morphogenic protein 7 (BMP7) in the mouse livers were quantified by RT-qPCR. BMP7-depleted MSCs were prepared and applied in this model, and compared to MSCs. Results: Liver fibrosis, portal hypertension and sodium retention that were developed by $\mathrm{CCl}_{4^{\prime}}$ were all significantly alleviated by MSCs transplantation, which decreased TGF $\beta 1$ levels and increased BMP7 levels in the injured liver. MSCs were found to express extremely high levels of BMP7. Knockdown of BMP7 in MSCs completely abolished the protective effect of MSCs against $\mathrm{CCl}_{4}$-induced cirrhosis. Conclusions: $\mathrm{MSCs}$ mitigate cirrhosis through their production of BMP7 against the fibrogenic effect of TGF $\beta 1$ in the injured liver.
\end{abstract}


Li et al.: MSCs Mitigate Cirrhosis through BMP7

\section{Introduction}

Mesenchymal stem cells (MSCs) have been initially identified in the bone marrow, and they are capable of extensively expanding in culture and differentiating into osteoblasts, chondrocytes, and adipocytes in responsive to corresponding stimulants. Moreover, MSCs have been shown to possess a particular property of immunosuppression in that it may alter immunity and inflammation during disease progresses [1]. Recent studies further demonstrate a potential role of MSCs in promoting hematopoietic engraftment and tissue repair [1-3]. When intravenously delivered, retention of MSCs mainly occurs in the lung [4, 5]. Therefore, orthotopic transplantation is needed to allow MSCs to properly function in organs other than the lung [6].

Hepatic fibrosis is overly exuberant wound healing in which excessive connective tissue accumulates in the liver [7-10]. The extracellular matrix is overproduced, degraded deficiently, or combined. The common trigger for hepatic fibrosis is injury-induced chronic inflammation, leading to portal hypertension due to distortion of blood flow through the liver by the scarring, or cirrhosis that results in disruption of normal hepatic architecture and liver dysfunction [7-10].

The current approaches to study the molecular mechanisms underlying the pathogenesis of cirrhosis often rely on the use of animal model [11-16]. So far, the most commonly used methods to induce chronic liver damage in mice are intragastric, intraperitoneal or subcutaneous administration of carbon tetrachloride $\left(\mathrm{CCl}_{4}\right)$ or other hepatotoxins, among which intraperitoneal injection of $\mathrm{CCl}_{4}$ has advantages to be low toxic to mice (least lethal) and reproducible induction of cirrhosis, although the levels of cirrhosis by this method may be not as pronounced as some other methods [11-16].

The cellular regulation of the development of hepatic fibrosis includes signaling molecules and receptors responsible for inflammatory cell recruitment, hepatocyte death and survival factors, and modulators of epithelial-mesenchymal transition (EMT), which plays an important role in the progression of fibrosis in the liver [17]. Major regulators of EMT in the liver are two multifunctional growth factors from transforming growth factor $\beta$ (TGF $\beta$ ) superfamily [18-20], bone morphogenic protein 7 (BMP7) and TGF $\beta 1$. While TGF $\beta 1$ is a well-established fibrotic inducer to promote transdifferentiation of hepatic stellate cells into myofibroblasts, BMP7 reverses EMT by directly counteracting TGF $\beta 1$-induced Smaddependent cell signaling [21-25]. Such antagonism coordinates the repair of the injured liver [26-30].

However, although transplantation of MSCs has therapeutic effects on various diseases, its effect on cirrhosis as well as the underlying mechanisms remains largely unknown. Here we examined the effects of MSCs on $\mathrm{CCl}_{4}$-induced cirrhosis in mice, and specifically evaluated the potential of BMP7 produced by MSCs.

\section{Materials and Methods}

\section{MSCs isolation, culturing, differentiation and transduction}

The MSCs were isolated and grown in culture as has been described previously $[3,6]$. Briefly, plugs of marrow from five 12-week-old male C57BL/6 mice (about 30g body weight) were dispersed in Dulbecco's Modification of Eagle's Medium (DMEM, Life technologies, San Jose, CA, USA) and then centrifuged at $900 \mathrm{~g}$ for $5 \mathrm{~min}$. The pellets were re-suspended and plated at $10^{5} \mathrm{cells} / \mathrm{cm}^{2}$ in DMEM containing $10 \%$ FBS. After 10 passages' selection of attached cells, the cells were sorted for Stro-1 (Becton-Dickinson Biosciences, San Jose, CA, USA) by flow cytometry to get rid of contaminating cells. A positive clone was selected after subjection to chondrogenetic, osteogenic, and adipogenic differentiation assays to confirm phenotype. For chondrogenetic induction, $2.5 \times 10^{5} \mathrm{MSC}$ were induced with $5 \mathrm{ml}$ chondrogenetic induction medium containing $10 \mu \mathrm{g}$ TGF $\beta 1$ (R\&D System, Los Angeles, CA,USA), 50 $\mathrm{g}$ insulin growth factor 1 (R\&D System), and $2 \mathrm{mg} / \mathrm{mL}$ dexamethasone (DMSO, Sigma-Aldrich, St. Louis, MO, USA) followed by centrifugation at $500 \mathrm{~g}$ for 
Li et al.: MSCs Mitigate Cirrhosis through BMP7

$5 \mathrm{~min}$. The cell pellets were maintained in the chondrogenetic induction medium for 14 days and subjected to Alcian blue staining. For osteogenic induction, cells were digested and seeded onto a 24-well plate at a density of $10^{4}$ cells/well, and then maintained in osteogenic induction medium containing 10nM Vitamin D3 (Sigma-Aldrich) and 10mM $\beta$-phosphoglycerol and $0.1 \mu \mathrm{M}$ DMSO for 14 days and were subjected to Von kossa staining. For adipogenic induction, cells were digested and seeded onto a 24 -well plate at a density of $10^{4}$ cells/well, and then maintained in the adipogenic induction medium containing 0.5mM 3-isobutyl1-methylxanthine (IBMX), $200 \mu \mathrm{M}$ indomethacin, $10 \mu \mathrm{M}$ insulin and $1 \mu \mathrm{M}$ DMSO for 14 days and subjected to Oil red 0 staining. For knock-down of BMP7 in MSCs, recombinant lentiviruses expressing either short hairpin small interfering RNA for BMP7, or scrambled sequence, were used to transduce MSCs at MOI 100 with nearly 100\% infection efficiency, resulting in MSCs-shBMP7 and MSCs-scr, respectively.

\section{Animal manipulations}

All animal procedures were conducted according to the guidelines for the care and use of laboratory animals approved by 302 Hospital of PLA. C57BL/6 mice (Charles River Laboratories, China) of 10 weeks of age were given free access to tap water and pelleted mouse diet. During cirrhosis induction, the mice were provided with $0.3 \mathrm{~g} / \mathrm{L}$ phenobarbital in drinking water to enhance $\mathrm{CCl}_{4}$ hepatotoxicity. For $\mathrm{MSCs}$ transplantation, MSCs $\left(10^{7}\right)$ were directly injected into the parenchyma of the liver at six spots.

\section{Induction of cirrhosis in mice}

Cirrhosis was induced by $\mathrm{CCl}_{4}$ intraperitoneal administration. Briefly, $\mathrm{CCl}_{4}$ solution of $50 \%(\mathrm{v} / \mathrm{v}$ ) in paraffin oil (Sigma-Aldrich, St. Louis, MO, USA) was administered at a dose of $2 \mathrm{ml}$ per kg body weight of the mice, by intraperitoneal injections twice a week for 8 weeks.

\section{Evaluation of liver fibrosis}

Liver samples were fixed in 10\% phosphate-buffered formalin, embedded in paraffin, and stained with the Sirius red staining technique. Fibrotic areas were counted on 200 random selected fields corresponding to approximately $6 \mathrm{~mm}^{2}$, using an unbiased counting frame, as has been described previously [14]. The percentage of fibrotic area was expressed as number of fibrotic fields divided by total fields, and then multiplied by 100 .

\section{Sodium balance}

The urine sodium concentration (UNa) was assayed by flame photometry (Roika 2000, Roika, UK), and renal sodium excretion (UNaV) was calculated with the following formula: $\mathrm{UNaV}=\mathrm{UV} \times \mathrm{UNa}$, where UV is urine volume. The intake of sodium was assessed by measuring the amounts of food and water consumed. Sodium balance was calculated as ( $\mathrm{Na}+$ provided by food and water)-UNaV.

\section{Portal pressure}

After evaluation of sodium metabolism, under anesthesia, a PE-50 polyvinyl catheter was placed in the cecal vein. The other end of the catheter was connected to a highly sensitive transducer (ADInstruments Shanghai Trading Co. Shanghai, China) to assess portal pressure.

\section{Quantitative real-time PCR (RT-qPCR)}

RNA was extracted from the liver tissue or the cultured MSCs with Trizol (Invitrogen, St Louis, MO, USA), and then used for cDNA synthesis. RT-qPCR was performed in duplicates with QuantiTect SYBR Green PCR Kit (Qiagen). All primers were purchased from Qiagen. Values of genes were normalized against $\alpha$-tubulin and then compared to the controls.

\section{Statistical analysis}

Statistical analyses were performed with SPSS 19.0 software (SSPS Inc., Chicago, IL, USA). All data were statistically analyzed using one-way ANOVA with a Bonferoni correction. $\chi 2$ test with Fisher's correction was used for comparison of survival in each experimental group. All values are depicted as mean \pm standard deviation from 5 individuals and are considered significant if $\mathrm{p}<0.05$. 


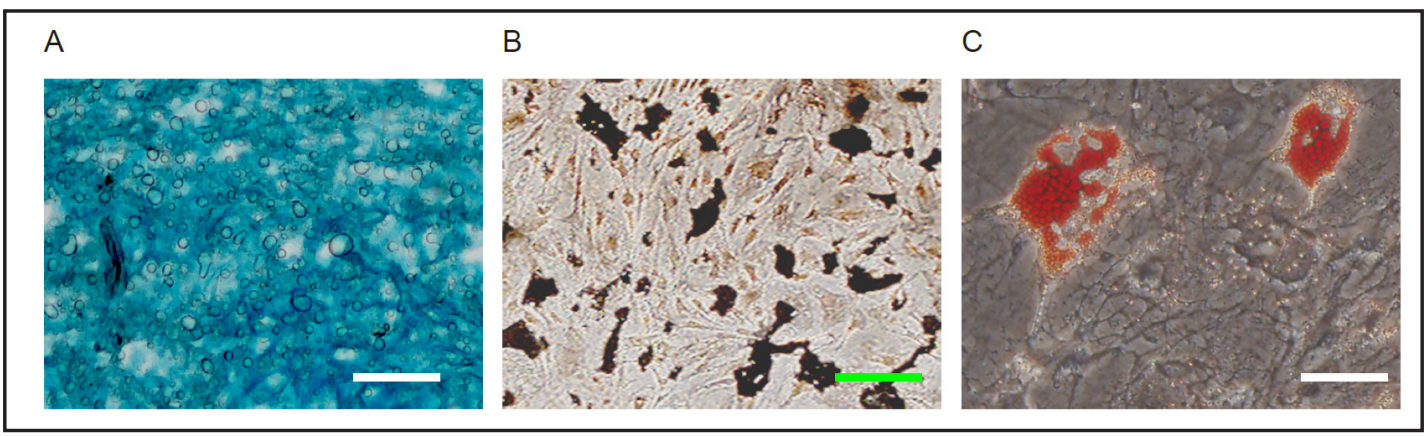

Fig. 1. MSCs culture and differentiation assays. (A-C) MSCs differentiation assay. (A) Alcian blue staining to evaluate chondrogenetic induction. (B) Von kossa staining to evaluate osteogenic induction. (C) Oil red 0 staining to evaluate adipogenic induction. Scale bars are $30 \mu \mathrm{m}$.

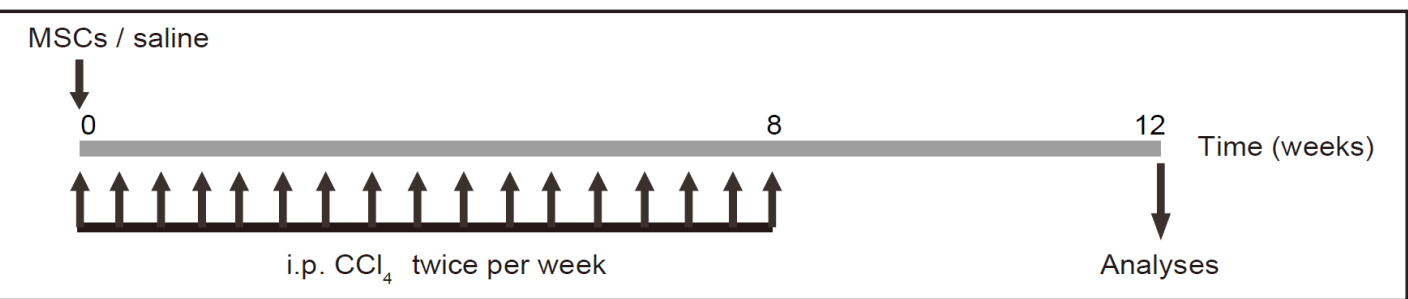

Fig. 2. Schematic of the model. Mice were first transplanted with MSCs of $10^{7}$, or control saline, directly into the liver, and then subjected to i.p. injection of $\mathrm{CCl}_{4}$ twice per week for 8 weeks. During this period, $0.3 \mathrm{~g} / \mathrm{L}$ phenobarbital was provided in drinking water to enhance $\mathrm{CCl}_{4}$ hepatotoxicity. Afterwards, the mice were kept for another 4 weeks, and then analyzed.

\section{Results}

Isolation, culturing and differentiation of primary mouse MSCs

Primary mouse MSCs were isolated from male C57BL/6 mice and expanded in culture. To confirm MSC phenotype, differentiation assays were performed, including Alcian blue staining to evaluate chondrogenetic induction (Fig. 1A), Von kossa staining to evaluate osteogenic induction (Fig. 1B), and Oil red 0 staining to evaluate adipogenic induction (Fig. 1C).

MSCs transplantation significantly alleviated the features of cirrhosis induced by CCl4

In order to evaluate the effect of transplantation of MSCs on cirrhosis, we used a wellestablished $\mathrm{CCl}_{4}$-induced cirrhosis animal model. Mice were first transplanted with $10^{7} \mathrm{MSCs}$, or control saline, directly into the liver, and then subjected to i.p. injection of $\mathrm{CCl}_{4}$ twice per week for 8 weeks. During this period, $0.3 \mathrm{~g} / \mathrm{L}$ phenobarbital was provided in drinking water to enhance $\mathrm{CCl}_{4}$ hepatotoxicity. Afterwards, the mice were kept for another 4 weeks, and then analyzed (Fig. 2). Mice mortality at sacrifice was very limited in both groups (1 from 10 each), with or without MSCs transplantation.

At sacrifice, all mice presented features of cirrhosis. The fibrotic area at sacrifice was evaluated after Sirius red staining, showing that MSCs transplantation significantly decreased the percentage of the fibrotic area (Fig. 3A). Portal hypertension and sodium metabolism were also assessed, showing that MSCs transplantation significantly decreased the portal pressure (Fig. 3B), significantly improved sodium balance (Fig. 3C), probably through an increased sodium excretion (Fig. 3D). These data suggest that MSCs transplantation significantly inhibited the development of cirrhosis induced by $\mathrm{CCl}_{4}$.

MSCs transplantation increased BMP7 and decreased TGFB1 in the injured liver

TGF $\beta 1$ is a well-known fibrosis promoter, while BMP7 has a potential role in antagonizing the effect of TGF $\beta 1$ in fibrosis. Thus, we examined whether MSCs may affect the balance 
Fig. 3. MSCs transplantation significantly alleviated the features of cirrhosis induced by $\mathrm{CCl}_{4}$ (A) The fibrotic area at sacrifice was evaluated after Sirius red staining, shown by the percentage of the fibrotic area. (B) Portal pressure. (C) Sodium balance. (D) Sodium excretion. *: $\mathrm{p}<0.05$. $\mathrm{n}=9$. Statistics: one-way ANOVA with a Bonferoni correction.
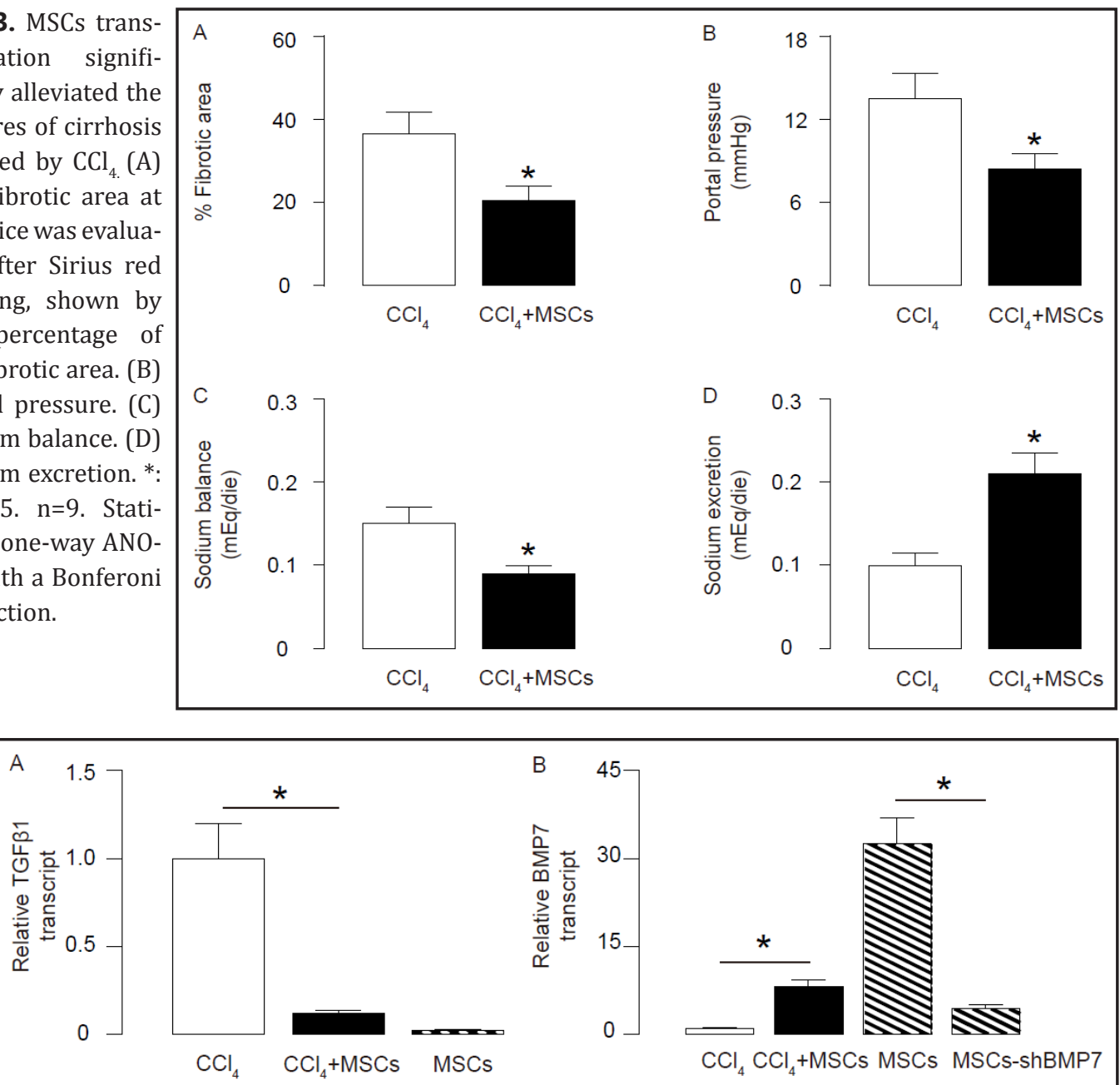

C

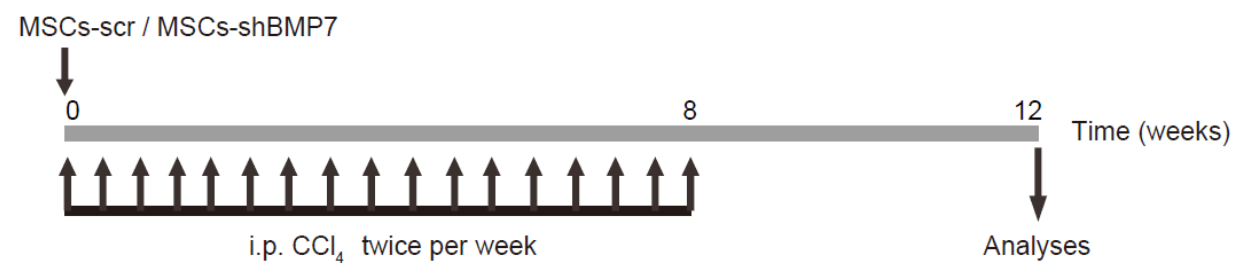

Fig. 4. MSCs transplantation increased BMP7 and decreased TGF $\beta 1$ in the injured liver. (A-B) Mouse livers at sacrifice (with/without MSCs) were examined for TGF $\beta 1$ (A) and BMP7 (B) transcripts, compared to the cultured MSCs, or MSC-shBMP7. (C) Schematic: Mice were first transplanted with MSCs-shBMP7 or MSCsscr of $10^{7}$ each, directly into the liver, and then subjected to i.p. injection of $\mathrm{CCl}_{4}$ twice per week for 8 weeks. During this period, $0.3 \mathrm{~g} / \mathrm{L}$ phenobarbital was provided in drinking water to enhance $\mathrm{CCl}_{4}$ hepatotoxicity. Afterwards, the mice were kept for another 4 weeks, and then analyzed. *: p<0.05. $n=9$. Statistics: one-way ANOVA with a Bonferoni correction.

of TGF $\beta 1$ and BMP7 in the injured liver to inhibit development of fibrosis. Mouse livers at sacrifice were thus analyzed for TGF $\beta 1$ and BMP7 transcripts, compared to the cultured MSCs. We found significantly decreased TGF $\beta 1$ (Fig. 4A) and significantly increased BMP7 (Fig. 4B) transcripts in the livers from the mice that had received MSCs transplantation, 


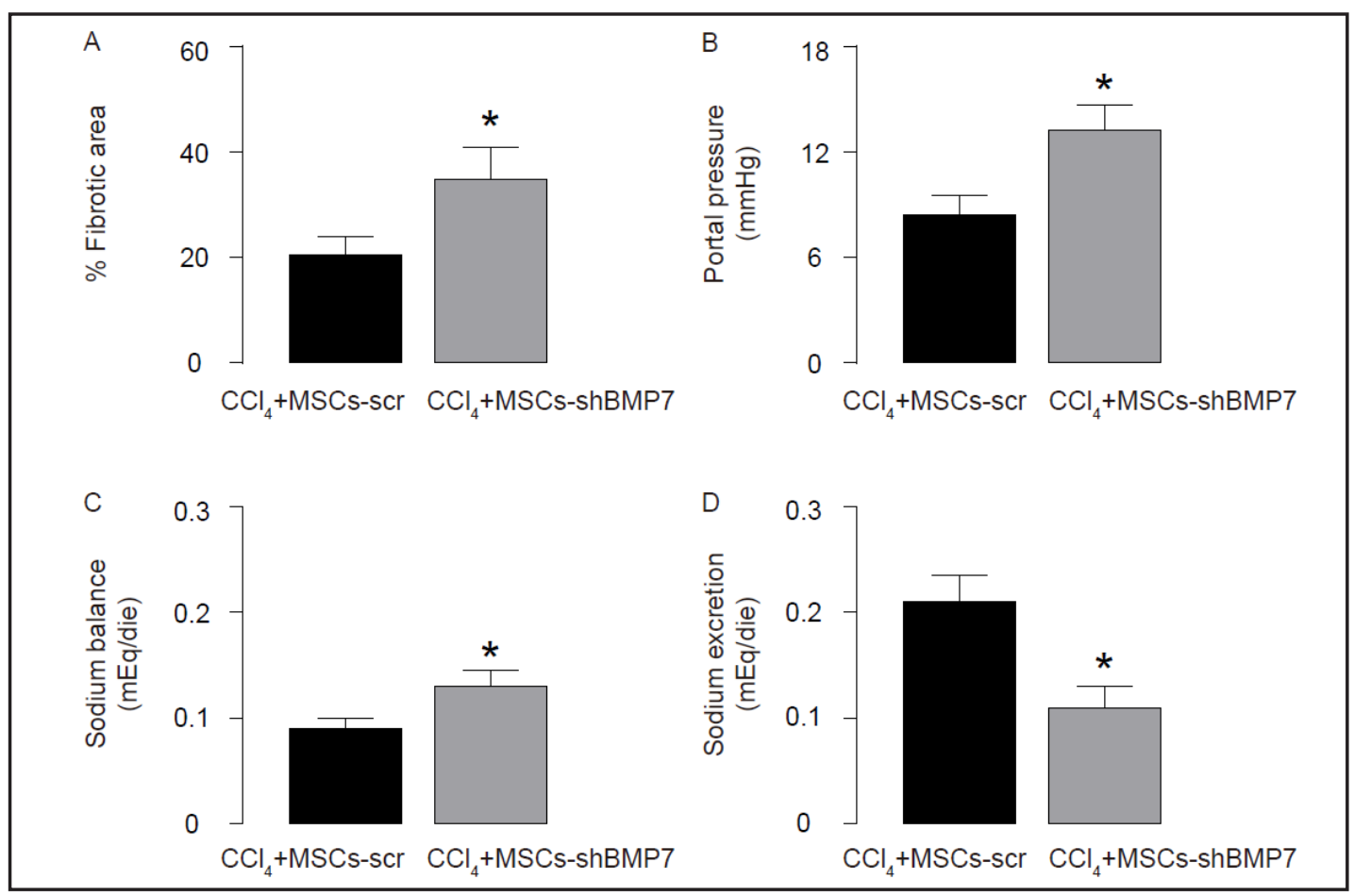

Fig. 5. Knock-down of BMP7 in MSCs abolished their anti-cirrhosis effect. (A) The fibrotic area at sacrifice was evaluated after Sirius red staining, shown by the percentage of the fibrotic area. (B) Portal pressure. (C) Sodium balance. (D) Sodium excretion. *: $\mathrm{p}<0.05 . \mathrm{n}=9$. Statistics: one-way ANOVA with a Bonferoni correction.

which supports our hypothesis. Moreover, it appeared that MSCs are a major source of BMP7 in the injured liver (Fig. 4B).

Knock-down of BMP7 in MSCs abolished its anti-cirrhosis effect

In order to find out whether BMP7 produced by MSCs is responsible for the inhibitory effect of MSCs transplantation on the development of cirrhosis, we inhibited the expression of BMP7 in MSCs using shRNA for BMP7 (MSCs-shBMP7). MSCs that were transduced with scrambled sequence (MSCs-scr) were used as controls, showing no difference with MSCs (not shown). The knock-down of BMP7 in MSCs was confirmed by examining BMP7 levels (Fig. 4B).

Then we transplanted these MSCs-shBMP7 or MSCs-scr into mice and induced cirrhosis with $\mathrm{CCl}_{4}$ (Fig. 4C). We found that knock-down of BMP7 in MSCs completely abolished its effects on fibrosis (Fig. 5A), portal pressure (Fig. 5B) and sodium metabolism (Fig. 5C-D), suggesting that the inhibitory effect of MSCs transplantation on the development of cirrhosis results from its production of BMP7, which may contradict the fibrotic effect of TGF $\beta 1$.

\section{Discussion}

$\mathrm{CCl}_{4}$-induced cirrhosis is a traditional cirrhosis model, which has been most commonly applied in the previous studies on chronic liver diseases [7-10]. Compared with genetically modified mouse models, $\mathrm{CCl}_{4}$-induced cirrhosis model has many merits, and best resembles human liver fibrotic diseases [11-16]. Among different ways of $\mathrm{CCl}_{4}$ administration including inhalation, subcutaneous injection and intraperitoneal injection, the latter has several advantages, e.g. fast development of cirrhosis, less toxicity and low lethality [11-16]. Although 
$\mathrm{CCl}_{4}$ by i.p may not be able to achieve highly severe cirrhosis and pronounced ascites, these parameters are not required for our study [11-16].

MSCs have been shown capable of regulating immunity and inflammation during disease progresses, and of promoting tissue repair [1-3]. In the current study, we injected MSCs directly into the parenchyma of the liver, since intravenous delivery of MSCs may suffer from pulmonary retention, resulting in poor cell perfusion into the liver [4-6].

We found that i.p injection of $\mathrm{CCl}_{4}$ successfully induced cirrhosis in all the mice, with typical histological changes of fibrosis, increased portal hypertension and sodium retention. However, MSCs substantially inhibited all these hallmarks for cirrhosis, suggesting that MSCs inhibits development of fibrosis. Since TGF $\beta 1$ is the most important activator for tissue fibrosis, and its fibrotic effect is often contradicted by BMP7 [18-20], we were prompted to examine whether MSCs may alter TGF $\beta 1$ and BMP7 levels in the injured liver. Our data confirmed our hypothesis, by showing a significant reverse of TGFß1-to-BMP7 ratio in the liver by MSCs transplantation, which may potentially result from the abundance of BMP7 produced by MSCs. We used transcripts of TGF $\beta 1$ and BMP7, rather than proteins for quantification, since there had been no reports about the post-translational controls of these two proteins.

In order to prove that BMP7 produced by MSCs is responsible for the inhibitory effect of MSCs transplantation on the development of cirrhosis, we inhibited the expression of BMP7 in MSCs by nearly 90\%. Interestingly, knock-down of BMP7 in MSCs completely abolished their effects on all the hallmarks of cirrhosis, which highlights a pivotal role of MSCs-derived BMP7 in the protection from cirrhosis.

In summary, our study not only demonstrates an inhibitory effect of MSCs transplantation on the development of cirrhosis, but also suggests that this protective effect results from the production of BMP7 by MSCs.

Finally, it should be taken into account that the mouse strain may affect the results in this study, and particularly may affect the responses of the mice to the $\mathrm{CCl}_{4}$ toxicity to influence the progression to and the level of fibrosis and cirrhosis. Future studies may be performed to evaluate this model in different strains, and in immunocompromised mice in responsive to transplantation of human MSCs.

\section{Acknowledgements}

This work was financially supported by Wang Baoen Foundation for Liver Fibrosis Research, NO 434510Z0A10 and National Natural Science Foundation of China, NO 81371799.

\section{Disclosure Statement}

The authors have declared that no competing interests exist.

\section{Reference}

1 Zipori D: Mesenchymal stem cells: Harnessing cell plasticity to tissue and organ repair. Blood Cells Mol Dis 2004;33:211-215.

2 Uccelli A, Moretta L, Pistoia V: Mesenchymal stem cells in health and disease. Nat Rev Immunol 2008;8:726-736.

3 Wang C, Chen J, Sun L, Liu Y: Tgf-beta signaling-dependent alleviation of dextran sulfate sodium-induced colitis by mesenchymal stem cell transplantation. Mol Biol Rep 2014;41:4977-4983.

-4 Fischer UM, Harting MT, Jimenez F, Monzon-Posadas WO, Xue H, Savitz SI, Laine GA, Cox CS Jr: Pulmonary passage is a major obstacle for intravenous stem cell delivery: The pulmonary first-pass effect. Stem Cells Dev 2009;18:683-692. 
5 Song X, Xie S, Lu K, Wang C: Mesenchymal stem cells alleviate experimental asthma by inducing polarization of alveolar macrophages. Inflammation 2014, Doi 10.1007/s10753-014-9954-6.

6 Cao X, Han ZB, Zhao H, Liu Q: Transplantation of mesenchymal stem cells recruits trophic macrophages to induce pancreatic beta cell regeneration in diabetic mice. Int J Biochem Cell Biol 2014;53:372-379.

7 Hirschfield GM, Gershwin ME: The immunobiology and pathophysiology of primary biliary cirrhosis. Annu Rev Pathol 2013;8:303-330.

8 Invernizzi P, Selmi C, Gershwin ME: Update on primary biliary cirrhosis. Dig Liver Dis 2010;42:401-408.

-9 Selmi C, Lleo A, Pasini S, Zuin M, Gershwin ME: Innate immunity and primary biliary cirrhosis. Curr Mol Med 2009;9:45-51.

10 Tangkijvanich P, Yee HF Jr: Cirrhosis--can we reverse hepatic fibrosis? Eur J Surg Suppl 2002:100-112.

-11 Selmi C, Meda F, Kasangian A, Invernizzi P, Tian Z, Lian Z, Podda M, Gershwin ME: Experimental evidence on the immunopathogenesis of primary biliary cirrhosis. Cel Mol Immunol 2010;7:1-10.

12 Chuang YH, Ridgway WM, Ueno Y, Gershwin ME: Animal models of primary biliary cirrhosis. Clin Liver Dis 2008;12:333-347; ix.

13 Concepcion AR, Medina JF: Approaches to the pathogenesis of primary biliary cirrhosis through animal models. Clin Res Hepatol Gastroenterol 2012;36:21-28.

14 Tsuneyama K, Moritoki Y, Kikuchi K, Nakanuma Y: Pathological features of new animal models for primary biliary cirrhosis. Int J Hepatol 2012;2012:403954.

15 Leung PS, Yang GX, Dhirapong A, Tsuneyama K, Ridgway WM, Gershwin ME: Animal models of primary biliary cirrhosis: Materials and methods. Methods Mol Biol 2012;900:291-316.

16 Wang J, Yang GX, Tsuneyama K, Gershwin ME, Ridgway WM, Leung PS: Animal models of primary biliary cirrhosis. Semin Liver Dis 2014;34:285-296.

17 Baghy K, Iozzo RV, Kovalszky I: Decorin-tgfbeta axis in hepatic fibrosis and cirrhosis. J Histochem Cytochem 2012;60:262-268.

18 Massague J: Tgfbeta signalling in context. Nat Rev Mol Cell Biol 2012;13:616-630.

19 Lan HY, Chung AC: Transforming growth factor-beta and smads. Contrib Nephrol 2011;170:75-82.

20 Xiao X, Gaffar I, Guo P, Wiersch J, Fischbach S, Peirish L, Song Z, El-Gohary Y, Prasadan K, Shiota C, Gittes GK: M2 macrophages promote beta-cell proliferation by up-regulation of smad7. Proc Natl Acad Sci USA 2014;111:E1211-1220.

21 Liu G, Cheng J, Guan G, Jia Z: Renal lymph circulation blockage alters the epithelial cell phenotype and tubular integrity: Role of distinct regulation of bmp7 and tgf-beta/smads signaling pathway. Int Urol Nephrol 2014;46:1239-1246.

22 Khan I, Agarwal P, Thangjam GS, Radhesh R, Rao SG, Kondaiah P: Role of tgf-beta and bmp7 in the pathogenesis of oral submucous fibrosis. Growth Factors 2011;29:119-127.

-23 Buijs JT, Henriquez NV, van Overveld PG, van der Horst G, ten Dijke P, van der Pluijm G: Tgf-beta and bmp7 interactions in tumour progression and bone metastasis. Clin Exp Metastasis 2007;24:609-617.

-24 Wang S, Hirschberg R: Bmp7 antagonizes tgf-beta -dependent fibrogenesis in mesangial cells. Am J Physiol Renal Physiol 2003;284:F1006-1013.

-25 Shen B, Liu X, Fan Y, Qiu J: Macrophages regulate renal fibrosis through modulating tgfbeta superfamily signaling. Inflammation 2014;37:2076-2084.

-26 Wang S, Hyun J, Youn B, Jung Y: Hedgehog signaling regulates the repair response in mouse liver damaged by irradiation. Radiat Res 2013;179:69-75.

27 Zeisberg M, Yang C, Martino M, Duncan MB, Rieder F, Tanjore H, Kalluri R: Fibroblasts derive from hepatocytes in liver fibrosis via epithelial to mesenchymal transition. J Biol Chem 2007;282:23337-23347.

28 Tacke F, Gabele E, Bataille F, Schwabe RF, Hellerbrand C, Klebl F, Straub RH, Luedde T, Manns MP, Trautwein C, Brenner DA, Scholmerich J, Schnabl B: Bone morphogenetic protein 7 is elevated in patients with chronic liver disease and exerts fibrogenic effects on human hepatic stellate cells. Dig Dis Sci 2007;52:3404-3415.

29 Kinoshita K, Iimuro Y, Otogawa K, Saika S, Inagaki Y, Nakajima Y, Kawada N, Fujimoto J, Friedman SL, Ikeda K: Adenovirus-mediated expression of bmp-7 suppresses the development of liver fibrosis in rats. Gut 2007;56:706-714.

30 Sugimoto H, Yang C, LeBleu VS, Soubasakos MA, Giraldo M, Zeisberg M, Kalluri R: Bmp-7 functions as a novel hormone to facilitate liver regeneration. FASEB J 2007;21:256-264. 